\title{
RARE POSITIVE LABORATORY TESTS FOR THE PRESENCE OF INFLUENZA VIRUS A/H1N1-2009 IN MAY, JUNE, JULY, 2011, IN THE DISTRICTS OF KOŠICE I-IV AND SURROUNDINGS OF KOŠICE IN THE SLOVAK REPUBLIC RAPID COMMUNICATION
}

\author{
Jana Seligová', Anna Belyová1, Andrea Čulmanová2, Vladimír Oleár ${ }^{3}$, Lýdia Čislákováa
}

${ }^{1}$ Regional Public Health Authority in Košice, Košice, Slovak Republic

${ }^{2}$ Faculty of Medicine UPJŠ in Košice, Košice, Slovak Republic

${ }^{3}$ Slovak Medical University in Bratislava, Bratislava, Slovak Republic

\begin{abstract}
SUMMARY
Influenza illnesses and positive laboratory tests for the presence of influenza virus in recent years in the districts of Košice I-IV and surroundings have only occurred during the winter season. In May to July 2010 only one positive laboratory test for the presence of influenza virus A/H1N1-2009 was reported. In 2011, during the same period, a total of 29 positive laboratory tests were recorded for the presence of influenza virus A/H1N1-2009 in individuals with typical clinical symptoms of influenza. Of 29 clinical cases, 27 were diagnosed as influenza and 2 as SARl; 4 cases involved children.
\end{abstract}

Key words: influenza, virus, summer

Address for correspondence: J. Seligová, Regional Public Health Authority in Košice, Ipel'ská 1, 040 11, Košice, Slovak Republic. E-mail: ke.seligova@uvzsr.sk

\section{INTRODUCTION}

In the Slovak Republic (SR), influenza surveillance has been carried out regularly since 1954, when Slovakia joined the Global WHO influenza surveillance program. The aim is an early detection of epidemic or pandemic influenza and rapid containment and identification of new variants or subtypes of influenza virus in order to prepare relevant vaccines (1-2). Influenza season in the northern hemisphere is the period from October 1 to April 30 the following year. During this period has the highest incidence of acute respiratory infections, including influenza and influenza-like illnesses peaks and the majority of positive laboratory tests for the presence of influenza virus are reported (3) with no positive laboratory tests recorded during summer season (May-August). Laboratory tests for all cases of infection are not feasible (many patients do not seek medical treatment) and would be of no use for surveillance purposes. Biological material from patients is collected by first-contact physicians and doctors in hospitals, with relevant follow-up of cases requiring hospitalization (4). During the 2002/2003 influenza season in the Slovak Republic, biological material from patients with influenza was collected by sentinel physicians throughout the year to secure monitoring of influenza viruses circulating in the population.

\section{MATERIAL AND METHODS}

Influenza illnesses and positive laboratory tests for the presence of influenza virus in recent years in the districts of Košice I-IV and surrounding area have occurred only during the winter. In 2010, the first positive laboratory test for presence of influenza virus outside the influenza season was reported (A/H1N1-2009, July, positive travel history). In July 2011, at the end of the 2010/2011 influenza season, 29 positive laboratory tests for the presence of influenza virus A/H1N1-2009 were confirmed by real-time PCR method, along with 6 positive isolations of influenza virus on MDCK cell cultures. Nasopharyngeal swabs were collected from 26 patients, pharyngeal washings from 2 patients and nasal secretions for virological tests from one child. Swabs were put into containers with viral transport medium - medium 199 with $0.5 \%$ BSA and antibiotics Penicillin G, streptomycin, amphotericin, gentamicin (WHO 2002) supplied by the laboratory $(5,6)$. Mortality from influenza in that period was very low.

Laboratory tests in the districts of Košice I-IV and surroundings were carried out by the virological laboratory of the Regional Public Health Authority in Košice.

\section{RESULTS}

A total of 29 positive laboratory tests for the presence of influenza virus A/H1N1-2009 from biological material of patients with suspected influenza was recorded from May 52011 - July 31 2011, involving 10 cases in May, 9 in June and 10 in July (Table 1).

In total, 27 patients were diagnosed with influenza and 2, both males, with SARI (Severe Acute Respiratory Infection). The case of one 58 year old patient man was fatal: primary symptoms, 
Table 1. Influenza and SARI in the districts of Košice I-IV and surroundings of Košice May 1 to July 31, 2011, by month

\begin{tabular}{|l|c|c|c|c|c|c|c|c|}
\hline \multirow{2}{*}{ District/month } & \multicolumn{2}{|c|}{ May } & \multicolumn{2}{c|}{ June } & \multicolumn{2}{c|}{ July } & \multicolumn{2}{c|}{ Total } \\
\cline { 2 - 9 } & Flu & SARI & Flu & SARI & Flu & SARI & Flu & SARI \\
\hline Košice I-IV & 8 & 0 & 4 & 0 & 5 & 1 & 17 & 1 \\
\hline Košice surroundings & 2 & 0 & 4 & 1 & 4 & 0 & 10 & 1 \\
\hline Total & 10 & 0 & 8 & 1 & 9 & 1 & 27 & 2 \\
\hline
\end{tabular}

Table 2. Influenza and SARI in the districts of Košice I-IV and surroundings of Košice from May 1 to July 31, 201,1 by gender

\begin{tabular}{|c|c|c|c|c|c|c|}
\hline \multirow{2}{*}{ District/gender } & \multicolumn{2}{|c|}{ Men } & \multicolumn{2}{|c|}{ Women } & \multicolumn{2}{|c|}{ Total } \\
\hline & Flu & SARI & Flu & SARI & Flu & SARI \\
\hline Košice I-IV & 3 & 1 & 14 & 0 & 17 & 1 \\
\hline Košice surroundings & 4 & 1 & 6 & 0 & 10 & 1 \\
\hline Total & 7 & 2 & 20 & 0 & 27 & 2 \\
\hline
\end{tabular}

Table 3. Influenza and SARI in the districts of Košice I-IV and surroundings of Košice May 1 to July 31, 2011, by age group

\begin{tabular}{|c|c|c|c|c|c|c|}
\hline \multirow{2}{*}{ Age group/district } & \multicolumn{2}{|c|}{ Košice I-IV } & \multicolumn{2}{|c|}{ Košice surr. } & \multicolumn{2}{|c|}{ Total } \\
\hline & Flu & SARI & Flu & SARI & Flu & SARI \\
\hline 0 & 1 & 0 & 1 & 0 & 2 & 0 \\
\hline $1-4$ & 0 & 0 & 1 & 0 & 1 & 0 \\
\hline $5-9$ & 0 & 0 & 1 & 0 & 1 & 0 \\
\hline $10-14$ & 0 & 0 & 0 & 0 & 0 & 0 \\
\hline $15-19$ & 0 & 0 & 0 & 0 & 0 & 0 \\
\hline $20-24$ & 2 & 0 & 1 & 0 & 3 & 0 \\
\hline $25-34$ & 4 & 0 & 2 & 0 & 6 & 0 \\
\hline $35-44$ & 3 & 0 & 1 & 1 & 4 & 1 \\
\hline $45-54$ & 3 & 0 & 2 & 0 & 5 & 0 \\
\hline $55-64$ & 2 & 1 & 1 & 0 & 3 & 1 \\
\hline $65+$ & 2 & 0 & 0 & 0 & 2 & 0 \\
\hline Total & 17 & 1 & 10 & 1 & 27 & 2 \\
\hline
\end{tabular}

acute myocarditis, appeared July 52011 followed by death on July 142011 from cardiogenic shock. A nose-throat swab from real-time PCR was positive for the presence of influenza virus A/ H1N1-2009. Cases of disease by gender are presented in table 2.

The age breakdown of cases is presented in Table 3 .

\section{The Clinical Picture}

The clinical picture was dominated by high temperatures $40{ }^{\circ} \mathrm{C}$ ( 2 without temperature), fever, chills, headache, muscle or joint paints, dry irritating cough, bronchopneumonia, dehydration, 3 cases diarrhea, 3 cases urosepsis, 1 case precollapse state, 2 cases cardiogenic shock. In one case ARDS (acute respiratory distress syndrome). Influenza virus A/H1N1-2009 was confirmed by real-time PCR in all patients.

\section{Hospitalization}

Severe clinical course resulted in 20 hospitalizations, of which two cases were aged over 65 years. The majority of patients were aged 24-58 years and hospitalized at the University Hospital L. Pasteur in Košice : 8 patients at the Department of Infectology and Travel Medicine, 5 at the Pulmonary Clinic, 1 at the Department of Acute and Intensive Care, 2 at the Eastern Institute of Heart Disease and 4 in the Children's Faculty Hospital in Košice. Length of hospitalization varied from 3 to 38 days (mode 5 days, median 8.2 days). Maximum length, 38 days, of hospitalization was for a patient diagnosed with SARI. Occupational division of patients: 3 health workers (12\%), 3 pensioners, 1 maternity leave, 16 other occupations.

\section{Vaccination}

Of 29 patients, only 3 were vaccinated against influenza: a 39 year old health care worker vaccinated in September 2010 (taken 
ill May 17, 2011), a 27 year old male vaccinated in November 2010 (taken ill May 22, 2011) and a 23 year old woman vaccinated in September 2010 (taken ill July 12, 2011). Patients had no temperature but reported a slight headache and muscle pains at the beginning of the disease. A slight dry, irritating cough subsided within one month. Bronchopneumonia or pneumonia was not diagnosed, patients were hospitalised.

\section{DISCUSSION}

Since 2009, when the A/H1N1 influenza virus first appeared, there have been many cases of influenza infections with demonstrable A/H1N1-2009 pathogenesis. The positivity of laboratory tests for the presence of influenza virus A/H1N1-2009 in our districts during May, June and July have ranged 8 to 10 per month (and this only in those cases requiring medical help). From the high number of hospitalized patients we see that most cases have a severe clinical course. One recorded case was fatal. The largest number of cases, $15(55.6 \%)$ were in the 25 to 54 years age group. A total of 4 children (14.8\%) were taken ill, including an infant aged 11 days. During summer period influenza virus has not been detected in our region. Traditionally, after the winter influenza season epidemic there is decreased morbidity from influenza and influenza-like illness, following on from greatly decreased viral circulation.

Literature sources concerning tropical and subtropical countries show that laboratory confirmed influenza can occur throughout the year with one or two peaks per year. Yearly occurrence then leads to the fact that the cumulative impact is not clear, albeit with visible strain on the health service, school absences etc. as experienced during influenza epidemics (7). Sporadic outbreaks of disease in our regions are free of deeper impact on public health and do not therefore call for antiepidemic measures.

Vaccination, albeit at a low rate in our group, has proved effective and reduced severity of symptoms, mortality, complication and hospitalization rates in this group (8).

Our results pointed out the importance of influenza causative agents surveillance even during the low influenza occurence season.

\section{REFERENCES}

1. Beran J, Havlík J. Influenza. 2nd ed. Praha: Maxdorf; 2005. (In Czech.)

2. World Health Organization. WHO consultation on priority public health interventions before and during an influenza pandemic [Internet]. Geneva: WHO; 2004 [cited 2009 Dec 11]. Available from: http://www.afro.who. int/index.php?option $=$ com_docman\&task $=$ doc_download\&gid $=5116$.

3. M'ikanatha NM, Lynfield $\mathrm{R}$, van Beneden $\mathrm{C} \bar{A}$, de Valk H. Infectious disease surveillance. 1st ed. Massachusetts (USA): Blackwell Publishing; 2007.

4. Krištúfková Z, Oleár V, Štefkovičová M, Hudečková H, Seligová J, Ondicová M, et al. Harmonization of influenza morbidity monitoring in Slovakia with the EU. In: Abstracts of proceedings. Tuition Day of the NRC. Bratislava: ÚVZ SR; 2007. p. 18. (In Slovak.)

5. World Health Organization. WHO Manual on animal influenza diagnosis and surveillance. Geneva: WHO; 2002.

6. World Health Organization. WHO European quidance for influenza surveillance in humans. Copenhagen: WHO Regional Office for Europe; 2009.

7. World Health Organization. Influenza vaccines: recommendations for the use of inactived influenza vaccines and other preventive measures. Wkly Epidemiol Rec. 2000;75(35):281-8.

8. Nichol KL. Benefits of influenza vaccination among healthy and highrisk persons across the age spectrum. International Congress Series. 2004;1263:48-50.

Received October 27, 2011

Accepted in revised form November 27, 2011 